\title{
Les technopoles entre utopie et non-lieu de la mondialisation : le cas de Sophia-Antipolis
}

Jacques Araszkiewiez et Paul Rasse

\section{(2) OpenEdition}

Édition électronique

URL : http://journals.openedition.org/communicationorganisation/2950

DOI : 10.4000/communicationorganisation.2950

ISSN : $1775-3546$

Éditeur

Presses universitaires de Bordeaux

Édition imprimée

Date de publication : 2 octobre 2004

ISSN : 1168-5549

Référence électronique

Jacques Araszkiewiez et Paul Rasse, "Les technopoles entre utopie et non-lieu de la mondialisation : le cas de Sophia-Antipolis », Communication et organisation [En ligne], 25 | 2004, mis en ligne le 27 mars 2012, consulté le 01 mai 2019. URL : http://journals.openedition.org/

communicationorganisation/2950 ; DOI : 10.4000/communicationorganisation.2950

Ce document a été généré automatiquement le 1 mai 2019.

(c) Presses universitaires de Bordeaux 


\title{
Les technopoles entre utopie et non-lieu de la mondialisation : le cas de Sophia- Antipolis
}

\author{
Jacques Araszkiewiez et Paul Rasse
}

1 Cet article vient en premier lieu, si ce n'est conclure, du moins ponctuer le travail de recherche qui a été conduit pendant l'année 2002-2003 au sein de notre Laboratoire. Il s'agit d'une part d'une synthèse, d'autre part d'une mise à distance, et enfin d'une réflexion critique concernant tant l'organisation et la communication de la technopole de Sophia-Antipolis, que l'approche que nous avons pu conduire sur cet objet de recherche ${ }^{1}$. Une synthèse tout d'abord, qui conduira assurément le lecteur à se plonger dans l'énigmatique complexité de la technopole; une mise à distance ensuite, car le temps a déjà passé qui autorise un autre regard; une réflexion critique enfin, que nous permet cette nouvelle publication sur Sophia-Antipolis et la mondialisation.

En second lieu, nous proposons au lecteur, comme en atteste la signature conjointe, deux regards sur la technopole au regard du phénomène complexe dit de « glocalisation ». À la manière du sociologue anglais Bauman ${ }^{2}$, Paul Rasse décrit les acteurs de Sophia-Antipolis comme des élites de la mondialisation vivant dans des clos sécurisés paradisiaques en marge du monde. En complément, ce comportement est analysé au regard de la faillite d'une utopie sophipolitaine. Elle visait simultanément à établir « un mode de relations professionnelles différent qui bouscule la logique de la concurrence et du cloisonnement et pose en son cœur la coopération, le partage et la fécondation des savoirs ${ }^{3}$ » et un projet de vie au sein d'une technopole qui devait être une nouvelle ville grâce à laquelle les contacts informels générés par les activités culturelles, sportives, civiques auraient concouru à la fertilisation croisée. Le second, celui de Jacques Araszkiewiez, tente de rendre compte de ce que la technopole était supposée être, au regard de ce qu'elle est réellement, afin de comprendre sa réussite, mais également les limites définies par son mode de construction. 


\section{Aux origines de Sophia-Antipolis}

3 Le concept de technopole s'est imposé dans les années 1980 lorsque les acteurs de l'aménagement des territoires, technocrates et élus confrontés aux restructurations du tissu industriel ont dû se rendre à l'évidence de la globalisation. Les facilités de la communication, en accélérant et en généralisant les processus de division du travail à l'échelle de la planète, avaient entraîné la délocalisation d'activités industrielles traditionnelles qui, jusque-là faisaient l'excellence et la puissance des pays occidentaux, les obligeant à se repositionner dans le secteur tertiaire à haute valeur ajoutée. L'implantation, le regroupement d'entreprises mobilisant la recherche et les nouvelles technologies, devenait un bon moyen d'améliorer l'image de marque des régions en crise et de les redynamiser. Des expériences plus anciennes, la ZIRST de Meylan à Grenoble et Sophia-Antipolis sur la Côte d'Azur, avaient ouvert la voie. Bien sûr, il y avait aussi la fameuse Route 128 à Boston et la Silicon Valley dans la banlieue de San Francisco qui, loin d'être encore ce qu'elles sont devenues, laissaient cependant penser qu'un environnement naturel exceptionnel, allié à un environnement culturel fait de grandes universités, était propice à l'innovation technologique ${ }^{4}$. Un chercheur de l'École des mines amoureux de l'arrière-pays Niçois, Pierre Laffitte, en avaient eu l'intuition, vers la fin des années soixante :

4 «Les industries lourdes associées par tous, au xixe siècle, aux brumes et aux fumées de Pittsburgh, de Birmingham, de la Lorraine, de la Ruhr », correspondent à une période révolue, «le progrès et l'avenir ne dépendent plus désormais des matières pondéreuses, charbon ou minerais., mais de la matière grise, de la qualité de la formation, de la qualité de l'environnement $\aleph^{5}$. Pour ce faire, il rêvait d'une cité de la sagesse et de la connaissance regroupant vingt mille chercheurs. De fait, Sophia-Antipolis qu'il contribua activement à créer, porte les stigmates d'une Utopie dont on retrouve la trace dans les discours fondateurs ${ }^{6}$, mais au final, ce technopôle pour célèbre et exemplaire qu'il soit est une $\mathrm{u}-\mathrm{lopo}^{7}$. un non-lieu, paradisiaque et paradigmatique de la mondialisation ${ }^{8}$.

\section{Le mythe}

Sophia-Antipolis est une technopole mythique. Mais elle est également l'incarnation d'un mythe. Cette composition redoublée, diffractée et, on le verra, multipliée, est en fait bien sûr caractéristique de la structure des mythes. La genèse de Sophia-Antipolis est digne des grands mythes fondateurs. En effet, se trouvent corrélées dans cette fondation de manière indissociable et confuse trois dimensions : une origine exogène de la technopole à situer au-delà de l'Atlantique, une origine endogène propre à la Méditerranée et un principe d'auto-engendrement. Ces trois dimensions autorisent Pierre Laffitte, homme réseau alors sous-directeur de l'École des Mines, à se situer en France, comme prophète d'une révolution déjà en marche ailleurs, mais également comme représentant, si ce n'est comme garant, du génie, des valeurs d'une civilisation occidentale dont le berceau est méditerranéen. Symétriquement, la réalisation de la prophétie ne pourra qu'attester de la vérité du récit initial et de la clairvoyance du prophète. Tentons de clarifier cette triple origine. 


\section{Une origine exogène ou comment la première technopole européenne constitue l'incarnation d'un mythe}

6 La Silicon Valley ne s'est pas créée en un jour. Dans la région de San Francisco Bay, il a fallu presque cent ans de recherches, de transfert de technologies entre des universités américaines et des entreprises spécialisées, notamment dans les télécommunications, pour que quelques étudiants brillants parviennent à produire les innovations nécessaires au fonctionnement et à la commercialisation des premiers ordinateurs personnels, devenant ainsi les promoteurs de la seconde révolution informatique. Il a fallu en somme un siècle d'histoire avant que la Silicon Valley puisse apparaître sur la scène mondiale. Pourtant, ce qui n'est qu'un mythe aux États-Unis - la création ex-nihilo de la Silicon Valley - est une réalité en France. En une vingtaine d'années, Sophia-Antipolis va prendre corps, passant de l'état de vague projet au statut de technopole. Sur un plateau de garrigue ne présentant aucune infrastructure et encore moins de structures industrielles ou de recherches, va naître la première technopole européenne. En se construisant ainsi, Sophia-Antipolis atteste de la vérité du mythe ouest-américain de la Silicon Valley. Symétriquement, le développement de la Silicon Valley apparaît comme la preuve éclatante de la validité du choix de Sophia-Antipolis. On comprend dès lors le tropisme fort qui rattache Sophia-Antipolis aux États-Unis. Mais on comprend également que, face aux imitations du modèle - même si Sophia-Antipolis n'en constitue pas une reproduction puisque les projets de développement des deux technopoles ont été concomitants -, Martin Kenny et l'ensemble des auteurs soulignent dans Understanding Silicon Valley ${ }^{9} q u e$ le concept n'est pas si aisément transposable.

\section{Une origine endogène ou comment la technopole azuréenne se constitue en mythe}

7 L'implantation de Sophia-Antipolis sur la Côte d'Azur est justifiée dans une plaquette de présentation de 1972: "Qualité de l'environnement, lieux attirants, joie de vivre s'associent dans notre esprit aux rives de la Méditerranée, ce berceau de la civilisation occidentale, avec ses plaines, ses cités, ses collines à la mesure de l'homme, qu'il s'agisse de l'Attique, du Latium, de la Toscane ou de la Provence. Les géologues nous expliquent que c'est là un effet de l'orogenèse d'âge alpin, effet qui se retrouve dans une autre région attrayante pour les innovateurs qu'est la, Californie ${ }^{10}$ » Ainsi, le plateau de Valbonne est-il naturellement destiné à accueillir une technopole et cette création n'est finalement qu'un juste retour des choses, puisque les rives de la Méditerranée constituent le berceau de la civilisation occidentale.

\section{Le récit d'auto-engendrement}

Lors de la création de Sophia-Antipolis, la Silicon Valley n'existait pas : la vallée de San José était encore la capitale californienne de la culture de la prune. Et si les rives de la Méditerranée ont bien été le berceau de la civilisation occidentale, force est de reconnaître qu'un plateau de garrigue abandonné ne pouvait guère être considéré comme une trace du génie méditerranéen. Le récit du développement de Sophia-Antipolis est 
donc le récit révolutionnaire d'un auto-engendrement. Sophia-Antipolis naît donc en réalité de l'idée du futur. Elle ne s'inspire d'aucune référence.

La fondation de Sophia-Antipolis est la reformulation d'une première proposition - la génération spontanée de la Silicon Valley - et, en même temps, une attestation de sa vérité. Simultanément, elle est l'actualisation d'une seconde suggestion liée au génie méditerranéen. Elle est également une réalisation autonome, reliant de manière révolutionnaire passé et futur, articulant de manière originale la discontinuité propre aux révolutions à la continuité d'une civilisation. Ses trois propositions, en somme hétérogènes et ensemble probablement en contrariété, se renforcent mutuellement, elles se trouvent ainsi conciliées dans l'idée d'une identité propre à une civilisation et la cristalisation d'un ailleurs. Tel est le mystère de la première technopole européenne.

\section{L'utopie}

10 Le développement progressif de la région de San Francisco Bay aboutit dans la fin des années soixante à un foisonnement d'innovations technologiques et permet de constater a posteriori l'existence d'une fertilisation croisée (cross fertilisation), naturellement induite, entre entreprises et universités. Pour fonder Sophia-Antipolis, il est postulé a priori et symétriquement que le rassemblement de chercheurs, d'industriels et d'artistes (dont les esthéticiens nous assurent qu'ils préfigurent l'avenir) ne manquera pas de générer l'innovation technologique tant espérée. Lorsque le chien est content, il bat de la queue. Donc, si le chien bat de la queue, c'est qu'il est content. Tel est le syllogisme réducteur, l'apparente logique agissante au cœur de Sophia-Antipolis. À l'origine de Sophia-Antipolis, se trouve une forme de pensée magique et aporistique s'accompagnant d'une volonté de transfiguration de la réalité. Articulé au mythe, en contre-point de ses zones obscures, se développe un récit porteur d'utopies.

11 D'une part, le mythe est adapté. Située dans une région nécessairement proche de Paris, centralisation française oblige, la proposition dont Pierre Laffitte « d'installer le Quartier latin aux champs » se transforme progressivement en « Cité de la sagesse, de la Science et des techniques » située sur le plateau de Valbonne, au sein de la Côte d'azur. Bien que la référence à la Silicon Valley ne puisse être invoquée - la Silicon Valley n'existe pas en encore, Jean-Jacques Servant Schreiber n'a pas fondé ['Express et l'esprit Côte Ouest des États-Unis n'est connu que d'une élite-, ce changement de localisation renforce la consistance de l'axe Méditerranée-Californie.

D'autre part, le mythe est expliqué. L'utopie devient agissante. Elle est vendue aux décideurs. Sophia-Antipolis ne correspond pas seulement à l'aménagement d'un territoire. Il s'agit de construire une nouvelle ville entièrement construite et organisée pour permettre la fertilisation croisée : "Sophia-Antipolis, citée vouée à la créativité, conçue pour la favoriser, créée de toutes pièces dans un site préservé où la nature garde ses droits, s'ordonnera autour d'un cœur, l'agora antique transposée en placette de village provençal. À l'ombre des platanes, l'on y échangera des idées, on y créera des entreprises et des emplois, on y tentera de concevoir le progrès sous tous ses aspects et de la mettre en œuvre. ${ }^{11}$ ». À Sophia-Antipolis, la vie devait donc s'ordonner autour d'une place centrale censée regrouper les services communs (hôtellerie, restauration, banque, poste, pharmacie, librairie, théâtre, cinéma, salles d'exposition et de séminaires, crèches...). Les services culturels devaient êtres regroupés dans une fondation bordant la 
place Sophie Laffitte, elle aurait dû être le lieu de toutes les rencontres, de communication et de partage des savoirs, le cœur de la cité et le signe de sa supériorité intellectuelle.

Parallèlement à ce protêt de mise en place de conditions propices, les grands utopistes sont convoqués pour justifier l'idée de fertilisation croisée : "L'idée d'une concentration intellectuelle hors des grandes villes présentait une convergence avec l'utopie de Thomas Moore, les projets des sains-simoniens ou l'aphorisme de l'humoriste Alphonse Allais : « Il faut construire des villes à la campagne $»^{12} »$. Ce n'est pas par hasard que Thomas More (1480-1535), quand il forge le mot « utopie », imagine une île qui est nulle part «U-topia » et en même temps, en raison de cette incertitude, possible partout. Pour que le rêve utopiste puisse se déployer pleinement, il faut un lieu improbable, un espace vierge, isolé du vieux monde malade afin d'éviter toute contamination et en même temps relié à lui par des canaux de communication conséquents, mais dont les flux sont contrôlés, car les utopies se veulent exemplaires pour le reste de l'humanité et donc en relation avec elle. Aussi l'iconographie des premières éditions d'Utopia, celle de Bâle notamment, publiée en 1518, montre une île reliée au continent par deux ponts et un port. De même dans «la Cité du Soleil », Tommaso Campanella décrit une ville protégée par sept enceintes et dont quatre portes contrôlent l'accès. Ce souci de créer des zones édéniques protégées, isolées des vacarmes et des violences du monde extérieur, mais liées à lui par des autoroutes réelles ou virtuelles, à haut débits, sécurisées, où s'épanouiraient des modes de vies pacifiées, libres à condition de se conformer strictement aux contraintes réglementaires du lieu, est une des caractéristiques de ces nouvelles villes où se rassemblent les élites mondiales, auquel, comme nous le verrons, s'apparente par bien des aspects le technopôle de Sophia-Antipolis.

\section{La fertilisation croisée}

La technopole de Sophia-Antipolis a été conçue comme une machine communicante et comme une machine à transmettre, des conditions optimales de communication étant supposées favorables à la meilleure transmission possible. En retour, la transmission, bien que limitée dans un premier temps aux corps élus, devait voir ses effets se communiquer au monde entier. En ce sens l'utopie portée par les technopoles était bien un précurseur de la mondialisation. Afin de créer les conditions nécessaires à la communication comme à la transmission, l'organisation de la technopole devait donc être idéale, c'est-à-dire dominée par la raison. «L'administration des choses devra remplacer le gouvernement des personnes " prônait Saint Simon; la plupart des projets utopiques s'efforcent d'imaginer des formes d'organisation de la vie sociale qui ne dépendrait plus d'un pouvoir pyramidal, mais d'un système de règles rationnelles s'imposant à tous par leur évidence, en faisant appel à la raison de chacun, de façon à garantir la dynamique et l'harmonie de la communauté utopique.

15 Mais l'incarnation d'une telle rationalité ne pouvait être que problématique. Le développement de la communication entre les corps constitués suppose une totale transparence de l'organisation, une transparence telle qu'elle exigerait l'effacement de traces laissées par l'organisation. Pour que la communication puisse être optimale, il faut en somme que l'organisation qui permet la co-présence des corps constitués disparaisse. Or, s'il est déjà difficile d'imaginer une organisation, même dominée par la raison, susceptible de répondre à une telle contrainte, il lui faut encore répondre à une 
contrainte totalement opposée. La transmission ne se fonde que sur l'obstacle, sur la résistance à une communication généralisée. Une telle résistance ne se développe que lorsque l'organisation est opaque, et qu'elle assure la distance réelle et symbolique nécessaire au fonctionnement des corps constitués.

Ainsi l'organisation de la technopole devait être totalement transparente pour favoriser la communication et nécessairement opaque pour favoriser la transmission. À cette organisation idéale et impossible de la citée pilotée par des scientifiques, on substitua bien vite une organisation classique déterminée par son adaptation au contexte local. Parallèlement, au concept de fertilisation croisée, se substitua rapidement le concept de knowledge management. L'un était fondé sur la création et la diffusion de savoir, l'autre est basé sur la possession et la rétention de ces mêmes savoirs. Le premier était utopique, le second est pragmatique. L'impossibilité de réaliser l'organisation idéale supposée par la fertilisation croisée s'est donc accompagnée de la mise en place d'une organisation réelle et d'un changement de perspective, d'autant plus évident que le concept de knowledge management ne s'applique pas en priorité aux technopoles mais aux entreprises. La mesure de cette torsion et de ses effets n'a pas encore été pleinement mesurée, toutefois, avant de s'y exercer, il faut souligner qu'ils sont d'autant plus subis que l'utopie portée par la technopole s'est entre-temps incarnée.

17 Le dispositif utopique, de ville idéale, n'est pensé que pour tenter de pallier à ce qui fait défaut à Sophia-Antipolis, une histoire. Dans Transmettre, Régis Debray oppose la communication comme transport dans l'espace à la transmission comme transport dans le temps : «La première est ponctuelle et synchronisante, c'est une trame : un réseau de communication relie surtout des contemporains (un émetteur à un récepteur simultanément présent aux deux bouts de la ligne). La seconde est diachronique et cheminante, c'est une trame, plus un drame : elle fait le lien entre les morts et les vivants, le plus souvent en l'absence physique des 'émetteurs ${ }^{13}$ ». L'opposition entre communication et transmission rend immédiatement saillant le paradoxe constitutif de l'identité Sophipolitaine. Quel est-il ? Celui de prétendre articuler la dimension spatiale caractéristique de la communication avec la dimension temporelle caractéristique de la transmission grâce à une organisation idéale. Ce paradoxe serait donc de penser qu'une communication nécessairement in praesentia pourrait renforcer une transmission pourtant le plus souvent in absentia. Le discours utopique devient alors le moyen de pallier ce paradoxe. Il offre à Sophia-Antipolis l'avenir qui permettra de construire de construire le plus rapidement possible une histoire. Mais simultanément, l'articulation du mythe et d'un discours utopique performatif va plonger Sophia-Antipolis dans une anhistoricité permanente. Sophia-Antipolis ne sera en somme qu'un curseur se déplaçant en permanence au présent avec l'idée de modernité.

\section{La performance de la proposition}

\section{Un bilan économique contrasté}

18 Les créateurs, financiers, utilisateurs, évaluateurs de la technopole en conviennent, Sophia-Antipolis est incontestablement une réussite. Les objectifs de Pierre Laffitte qui faisait sourire l'intelligentsia parisienne : créer une cité de 20000 chercheurs là où il n'y avait qu'un désert de garrigue, ont été atteints en 1998. Malgré la crise économique ambiante, les vagues de licenciements et quelques départs très remarqués de grandes 
entreprises, le parc a suivi une croissance assez régulière pour dépasser en 2000, le seuil de 24000 emplois, gérés par 1227 entreprises, auxquels s'ajoutent les 10000 à 15000 salariés sous-traitants ; le tout réparti sur 23000 ha appartenant à cinq communes. La technopole ambitionne à présent d'atteindre le nombre de 40000 emplois, et pour cela, d'étendre le site aux communes voisines.

La réussite, en matière d'urbanisation, d'architecture et d'environnement, est elle aussi patente. Sophia-Antipolis est aux antipodes des zones industrielles et commerciales, ces étendues sinistres de hangars préfabriqués, standardisés, qui se multiplient à la périphérie des villes. La plupart des entreprises, et même, assez souvent, les collectivités, n'ont généralement pas lésiné pour construire des bâtiments transparents de bonne qualité, non clôturés, mais dispersés dans des parcs paysagers, etc. En l'apparence donc, l'utopie est devenue réalité, les caractéristiques du projet initial ont été respectées, mais, en apparence seulement. Car la technopole est un échec au plan de l'urbanité, de la fertilisation croisée et du développement culturel qui devaient en faire la Cité de la Sagesse. Sophia-Antipolis souffre de nombreux déséquilibres et de la faiblesse du pôle universitaire.

Si l'enquête conduite par Djamila El Idrissi et Valérie Hauch montre qu'une dynamique des clubs est présente, l'enquête de veille territoriale de Yann Bertacchini révèle que les clusters formés traduisent l'isolement des entreprises au sein du territoire sophipolitain et que les liens virtuels entre les entreprises et l'Université de Nice-Sophia-Antipolis sont souvent fragiles, et parfois même inexistants. De même, au-delà de leurs limites, l'enquête de Claudine Batazzi-Alexis qui peine à identifier un mode de management sophipolitain humaniste ou l'enquête de Edwige Vercnocke qui établit le peu de médiatisation des dirigeants à Sophia-Antipolis, sont révélatrices de dysfonctionnements ${ }^{14}$. La technopole de Sophia-Antipolis, bien qu'elle soit une technopole thématique centrée sur les télécommunications, les biotechnologies et l'informatique n'arrive pas à trouver d'unité. Les entreprises vivent essentiellement repliées sur elles-mêmes, privilégiant les échanges avec leur siège, leurs filiales, leur clientèle, dispersées de par le monde. La soustraitance pourrait être la seule façon pour elles de s'ouvrir sur l'extérieur, sur d'autres entreprises du site. Mais, d'après une enquête réalisée par la Chambre de Commerce et de l'Industrie en 1988, $10 \%$ seulement de cette sous-traitance se fait au plan local ou départemental. S'il semble que depuis les choses se soient améliorées à la suite des phénomènes d'essaimage et de la spécialisation d'entreprises locales, cela n'a encore été vérifié par aucune autre étude, et dans tous les cas, les rapports qui s'établissent sont alors au mieux des rapports formels de subordination, loin de la fameuse sociabilité cultivée et informelle que suppose la fertilisation croisée.

\section{Un investissement social limité}

21 Suivant la tripartition proposée par Jean-Thierry Maertens $\mathrm{s}^{15}$ en matière de rituel, SophiaAntipolis constitue une synthèse originale. La technopole reprend du système sauvage l'idée d'une communion avec la nature sur le lieu de travail, avec une préférence pour le logos dans l'échange (sur la placette, au centre de la technopole), idée qui se traduit également en termes d'aménagement par la nécessité de se déplacer avec un guide dans Sophia lorsqu'on s'y rend pour la première fois (très grande difficulté à se repérer malgré une abondante signalétique). Elle reprend du système barbare l'idée d'un lieu protégé, même s'il est en communication avec le reste du monde, et réservé à une élite. Elle 
reprend enfin du système civilisé l'idée d'une mise à disposition des savoirs constitués, d'un partage des bénéfices acquis pour l'ensemble de la société. Cette synthèse originale, articulée au principe de fertilisation croisée, devait garantir aux usagers une valeur ajoutée bien supérieure à la simple satisfaction de vivre sur la Côte d'Azur.

L'enquête semi qualitative menée auprès de 360 personnes travaillant sur la technopole ${ }^{16}$ révèle que le projet initial d'offrir un site exceptionnel susceptible d'attirer les meilleures compétences, fonctionne parfaitement. $45 \%$ des salariés sont des cadres et ce rapport atteint $72 \%$ dans le secteur des sciences et technologies de l'information. La plupart des salariés sont arrivées à Sophia-Antipolis pour des mobiles liés à l'emploi, (mutations ou embauches), ils sont généralement très satisfaits d'y travailler pour des raisons extraprofessionnelles liées à l'image de marque du site et à l'environnement qu'il réserve à ses employés. Pour autant, le site n'est pas vraiment habité. Des zones de logements ont été réalisées conformément au projet initial, mais les constructions sont de médiocre qualité et les conditions de vie quotidiennes y demeurent difficiles. En raison de l'isolement, des carences en équipements collectifs et en lieux de rencontre, les résidants y sont surtout des personnes à revenu modeste, souvent trop peu qualifiées pour êtres employées sur le site, si bien qu'ils sont contraints d'aller travailler à l'extérieur. Au contraire, les cadres dont les moyens financiers sont supérieurs préfèrent habiter dans les villes et villages alentour. Matin et soir, les uns et les autres se croisent en d'interminables embouteillages.

Ainsi, 95 \% des personnes interviewées n'habitent pas Sophia-Antipolis, mais en moyenne à 24 minutes ou 19 kilomètres de là, dans les communes alentours principalement Amibes et Nice, mais aussi Grasse, Cannes, Mougins, Cagnes sur mer... Et pourtant en dépit des difficultés d'accès, de la beauté du lieu, de son prestige, $8 \%$ seulement des personnes qui n'habitent pas sur le site voudrait y résider.

Notre enquête sur les modes de vie sophipolitains montre qu'au mieux, un salarié sur deux à la possibilité de rencontrer des travailleurs d'autres entreprises en dehors des relations professionnelles formelles imposées par ses activités et son statut professionnel. Mais l'idée de fertilisation croisée suppose de participer à un réseau de relations sociales dense, d'entretenir de nombreux liens avec des personnes extérieures à l'entreprise. Cela ne concerne plus alors que $16 \%$ des sophipolitains qui multiplient les opportunités de rencontre, à l'occasion des repas, d'activités sportives de cultures ou de loisir, et de façon plus marginale, en participant aux clubs et associations du site.

\section{Un non-lieu de la mondialisation}

Le mythe, l'utopie, l'image très investie de la technopole (jusqu'à produire une concurrence très importante entre les différents organismes chargés de veiller à son développement) lui ont donné une stature internationale. Son positionnement avantgardiste lui a par ailleurs peut être apporté l'avance nécessaire pour que la réalité et l'image convergent. La présence de grandes entreprises comme Amadeus, de structures internationales comme le W3C Consortium, la présence renforcée de l'Université de Nice Sophia-Antipolis et de nombreuses écoles et centres de recherche permettent de penser que la masse critique nécessaire est atteinte pour garantir les développements futurs de la technopole.

Pourtant, la Cité de la Sagesse n'est en définitive qu'un non-lieu de la mondialisation, une cité prestigieuse réservée aux nantis, les cadres privilégiés des grandes multinationales 
travaillant dans des domaines de pointe. Le sociologue anglais Bauman explique que, dans le cadre de la globalisation, les élites de la mondialisation ont pris l'habitude de s'installer dans des endroits paradisiaques, des lieux prestigieux où les meilleures conditions d'existence leur sont garanties. Ils vivent là, dans ces clos sécurisés, en contact avec le reste du monde, mais isolés des habitants alentour qu'ils ne fréquentent pas, dans un même confort exceptionnel et standardisé, sécurisé, en apparence ouverts, qui leur permettent de conserver les mêmes modes de vie sous-toutes les latitudes, d'être partout chez eux, et en même temps de s'attacher nulle part. Aussi sont-ils, comme les investisseurs, extrêmement mobiles, à l'affût des meilleures opportunités, prêts à se déplacer dès que leurs conditions d'existence privilégiée sont menacées, ou simplement parce qu'ils peuvent espérer en gagner de meilleures encore. Ces membres de la nouvelle aristocratie mondiale présentent bien des similitudes et pourtant n'ont rien à voir avec les U-Topos. Pour l'instant, le rapport prestige / qualité de vie / prix de la technopole de Sophia-Antipolis est sans doute encore l'un meilleur qui soit, cela en explique son succès, en dépit de la faillite du projet utopique... Souhaitons qu'on n'en restera pas la. D'autres élites, les plus importantes pour l'avenir sans doute, des chercheurs uniquement préoccupés de la réussite de leur projet scientifique et des conditions directes qui la déterminent, ne resteront pas nécessairement ou ne viendront plus à Sophia-Antipolis, si l'image renvoyée ne correspond pas à l'activité scientifique réelle attendue. Le pari du processus, de la vitesse de réponse, du dynamisme, n'est jamais gagné par avance. Et la difficulté ne pourra aller progressivement qu'en augmentant, dans la mesure où le coût humain et financier de la recherche fondamentale, cette recherche si déterminante en amont pour l'innovation technologique située en aval, ne manquera pas, lui aussi d'augmenter, jusqu'à provoquer une concentration massive des pôles de recherches. Sophia-Antipolis saura-t-elle en fixer quelques-uns?

\section{NOTES}

1. Cf. L'Héritage d'une utopie. Essai sur la communication et l'organisation de Sophia Antipolis, dir. Jaques AraszkiewieZ., Édisud, 2003.

2. Bauman Zygmunt, Le coût humain de la mondialisation, Hachette Littératures, 1999

3. Bourdin Sylvie, Genèse de Sophia-Antipolis : 1960-1974 ; L'efficacité des pratiques discursives, in l'Héritage d'une utopie, op. cit.

4. «Vers la lin des années 1960, raconte Pierre Laffite, la ville de San José était encore la capitale californienne de la prune. Toutefois, on savait qu'entre San José et San Francisco, le parc de l'Université de Stanford louait des hangars à des industriels attirés par le réservoir de compétences proches, ce qui installait une dynamique créative nouvelle. Laffite Pierre, Naissance d'une ville ? Sophia-Antipolis, Corps écrit n²9,PUF 1989, p. 13.

5. Sophia Antipolis, plaquette éditée par Savalor, Le BIAM et Armines, 1972, p. 2.

6. Voir Sfez Lucien, Prélace, Utopie 1, La fabrique de l'Utopie, Quaderni $N^{\circ} 40$ Sapientia, 1999/2000

7. Thomas More (1480-1535), quand il forge le mot « utopie ", imagine une île qui est nulle part «U-topia » et en même temps, en raison de cette incertitude, possible partout. 
8. Pour reprendre l'expression de Balandier. Balandier Georges, Le Grand Système, Fayard, 2001, p. 60.

9. Kenney Martin, Understanding Silicon, Stanford University Press, 2000.

10. Sophia Antipolis, plaquette, op. cit.

11. Sophia Antipolis, plaquette, op. cit.

12. Sophia Antipolis, plaquette, op. cit

13. Debray Régis, Transmettre, Odile Jacob, 1997, p. 17.

14. Articles publics dans L'Héritage d'une utopie. Essai sur la communication et l'organisation de Sophia . Antipolis dir. Jacques Araszkiewiez, Édisud, 2003.

15. Maertens Jean-Thierry, Ritologiques I. II. III. et V Le dessein sur la peau. Le corps sexionné, Le masque et le miroir. Dans la peau des autres. le jeu de la mort. Aubier, Paris, 1978.

16. L'enquête a été réalisée entre juin et septembre 2002 interrogées à la sortie des entreprises, sur les parkings et les trottoirs, en ayant soin de varier les lieux, les moments du lour et de la semaine. Nous nous sommes efforcés de cerner la vie quotidienne concernant tout particulièrement la sociabilité et les relations entre les salariés des entreprises, les chiffres détaillés ont été publiés dans Paul Rasse, Enquête statistique sur la sociabilité et la fertilisation croisée in Araszkiewiez Jacques, dir., l'Héritage d'une utopie, essai sur la communication et l'organisation de Sophia Antipolis, in Edisud 2003.

\section{RÉSUMÉS}

Cet article issu d'une recherche menée pendant l'année 2002-2003, vise à une réflexion critique concernant l'histoire, l'organisation et la communication de la technopole de Sophia-Antipolis, au regard du phénomène complexe dit de "glocalisation". Nous montrons comment, à ses origines la technopole qui devait concourir à la fertilisation croisée, fonctionne comme une utopie, et comment elle aboutit finalement à n'être qu'un non-lieu privilégié de la mondialisation réservée aux élites vivant dans des clos sécurisés paradisiaques en marge du monde. La comparaison entre ce que la technopole était supposée être, au regard de ce qu'elle est réellement, permet de comprendre sa réussite, mais également les limites définies par son mode de construction.

This article resulting from a research undertaken during the year 2002-2003, aims at a critical reflexion concerning the history, the organization and the communication of the technopolis of Sophia-Antipolis, in comparison with the complex phenomenon called of "glocalisalion ». We show how, at its origins the technopolis which was to contribute to the crossed fertilization, functions like an Utopia, and how it finally leads to be only one privileged withdrawal of case of the universalization reserved for the elites living in paradisiac protected fields in margin of the world. The comparison between what the technopolis was supposed to be, taking into consideration what it is really, makes it possible to understand its success, but also the limits defined by its method of construction. 


\section{AUTEURS}

\section{JACQUES ARASZKIEWIEZ}

Maître de Conférences en sciences de l'information et de la communication et directeur du département Information-Communication de l'IUT à l'Université de Nice Sophia Antipolis. Chercheur au laboratoire de Sciences de l'Information et de la Communication I3M (Milieux, médias médiation), il a dirigé le groupe de recherche sur la communication du technopole de Sophia Antipolis.

\section{PAUL RASSE}

Professeur des universités en sciences de l'Information et de la Communication, Directeur du laboratoire I3M à l'Université de Nice Sophia-Antipolis, et de la spécialité Médiation et d'ingénierie culturelle (année 2 de Master). 